\title{
Controle de Senecio madagascariensis e de Senecio brasiliensis pela utilização de pastejo com ovinos ${ }^{1}$
}

\author{
Adriana Lücke Stigger², Pablo Estima-Silva², Ana Carolina Barreto Coelho², Bianca \\ Lemos Santos ${ }^{2}$, Clairton Marcolongo-Pereira ${ }^{3}$, Franklin Riet-Correa ${ }^{4}$, Fabio Raphael \\ Pascoti Bruhn ${ }^{5}$ e Ana Lucia Schild ${ }^{6 *}$
}

\begin{abstract}
Stigger A.L., Estima-Silva P., Coelho A.C.B., Santos B.L., Marcolongo-Pereira C., Riet-Correa F., Bruhn F.R.P. \& Schild A.L. 2018. [Control of Senecio madagascariensis and Senecio brasiliensis by the use of sheep.] Controle de Senecio madagascariensis e de Senecio brasiliensis pela utilização de pastejo com ovinos. Pesquisa Veterinária Brasileira 38(1):29-36. Laboratório Regional de Diagnóstico, Faculdade de Veterinária, Universidade Federal de Pelotas, Campus Universitário s/n, Pelotas, RS 96010-900, Brazil. E-mail: alschild@terra.com.br

In order to test different technics to control Senecio madagascariensis, three experiments were carried out. In the first, 40 sheep were placed in an area of four hectares for 90 days, with medium/high levels of infestation by the plant. The area after this period was desiccated with glyphosate (Roundup ${ }^{\circledR}$ ) and seeded with Lotus corniculatus L., Trifolium repens, Medicago sativa and Festuca arundinacea Schreb. by direct seeding. After eight months, sheep returned to the area for another 90 days. The second experiment was conducted with 10 sheep grazing for 30 days and 60 days' rest, in three areas of 0.5 hectares each, with low, medium and high levels of $S$. madagascariensis infestation. The third experiment was carried out using drying, plowing and cultivated pasture (legumes and grasses) for three consecutive times without the use of sheep in an area infested by the plant. For the control of $S$. brasiliensis and other species of the genus, a fourth experiment was performed on a farm with history of intoxication by Senecio spp. in cattle. Eighty-six sheep were used in an area of 90 hectares for a year. The results of these experiments demonstrated that sheep consume and decrease the amount of $S$. madagascariensis in infested areas. Furthermore, it also indicated that $S$. madagascariensis to be efficiently controlled requires continuous grazing with at least four sheep per hectare. Practices as drying the pastures with herbicides, tillage and pasture planting can help eliminate the plant in long-term plan. In areas with $S$. brasiliensis infestation mowing can be an effective practice, mainly due to the high size of the plant, since it facilitates consumption by sheep.
\end{abstract}

INDEX TERMS: Poisonous plants, Senecio madagascariensis, Asteraceae, hepatotoxic plants, seneciosis, plant poisoning, cattle, toxicoses.

\footnotetext{
${ }^{1}$ Recebido em 9 de janeiro de 2017.

Aceito para publicação em 31 de março de 2017.

${ }^{2}$ Programa de Pós-Graduação em Veterinária, Faculdade de Veterinária (FV), Universidade Federal de Pelotas (UFPel), Campus Universitário s/n, Pelotas, RS 96015-560, Brasil.

${ }^{3}$ Faculdade de Veterinária, Centro Universitário Ritter dos Reis (UniRitter), Rua Orfanotrofio 555, Alto Teresópolis, Porto Alegre, RS 90840-440, Brasil.

${ }^{4}$ Hospital Veterinário, CSTR, Universidade Federal de Campina Grande (UFCG), Patos, PB 58700-000, Brasil.

${ }^{5}$ Departamento de Medicina Veterinária Preventiva, Faculdade de Veterinária, UFPel, Campus Universitário s/n, Pelotas, RS 96015-560, Brasil.

${ }^{6}$ Laboratório Regional de Diagnóstico, FV-UFPel, Campus Universitário s/n, Pelotas, RS 96015-560, Brasil. *Autor para correspondência: alschild@terra.com.br
}

RESUMO.- Com o objetivo de testar diferentes formas de controle de Senecio madagascariensis foram realizados três experimentos. No primeiro, 40 ovinos foram colocados em uma área de quatro hectares por 90 dias, com infestação média e alta por $S$. madagascariensis. A área após este período foi dessecada com glifosato (Roundup ${ }^{\circledR}$ ) e semeada com Lotus corniculatus L. (cornichão), Trifolium repens (trevo branco), Medicago sativa (alfafa) e Festuca arundinacea Schreb. (festuca) por plantio direto. Os ovinos, após oito meses, retornaram a área por mais 90 dias. 0 segundo experimento foi realizado com 10 ovinos em pastejo por 30 dias com 60 dias de descanso em três áreas de 0,5 
hectares cada uma, com infestação baixa, média e alta por S. madagascariensis. 0 terceiro experimento foi realizado utilizando-se dessecação, aração e plantio de pastagens (leguminosas e gramíneas) por três vezes consecutivas, sem utilização de ovinos em uma área invadida pela planta. Para o controle de $S$. brasiliensis e outras espécies do gênero, um quarto experimento foi realizado em uma propriedade rural com histórico de intoxicação por Senecio spp. em bovinos. Foram utilizados 86 ovinos, que permaneceram em uma área de 90 hectares durante um ano. Os resultados destes experimentos demonstraram que os ovinos consomem S. madagascariensis e diminuem a quantidade de planta em áreas infestadas. Por outro lado, evidenciou-se também que $S$. madagascariensis para ser controlado de forma eficiente necessita de pastejo contínuo com pelo menos quatro ovinos por ha. As práticas como dessecação com herbicidas, aração e plantio de pastagem podem auxiliar na eliminação da planta a longo prazo. Em áreas de infestação por $S$. brasiliensis a roçagem pode ser uma prática eficiente, principalmente pelo porte alto da planta, pois facilita o consumo pelos ovinos.

TERMOS DE INDEXAÇÃO: Plantas tóxicas, Senecio spp, Asteraceae, seneciose, intoxicação por plantas, ruminantes, toxicoses.

\section{INTRODUÇÃo}

Senecio spp. é sem dúvida uma das mais importantes plantas tóxicas da região Sul do Brasil para bovinos e a intoxicação nesta espécie tem sido amplamente estudada, especialmente no estado do Rio Grande do Sul devido a sua importância como causa de morte e prejuízos econômicos consideráveis (Barros et al. 1992, Karam et al. 2004, Grecco et al. 2010). A intoxicação tem sido descrita eventualmente também em ovinos (Ilha et al. 2001, Grecco et al 2011). Além das espécies do gênero conhecidas e descritas como causa de surtos como $S$. brasiliensis, $S$. heterotrix, S. selloi, $S$. oxyphyllus e S. tweediei (Méndez \& Riet-Correa 1993, Méndez \& Riet-Correa 2008), uma nova espécie, S. madagascariensis, foi identificada no Estado na década de 1990 (Matzenbacher \& Schneider 2008) e, posteriormente, surtos da intoxicação por esta espécie foram descritos em bovinos de leite e de corte (Cruz et al. 2010, Stigger et al. 2014).

S. madagascariensis é nativa da Ilha de Madagascar e África do Sul e foi introduzida de forma acidental em diversos países incluindo países da América do Sul como Uruguai e Argentina, além do Brasil (Cabrera 1963, Cabrera \& Ré 1965, Lombardo 1983, Villalba \& Fernández 2005). Esta espécie possui uma grande capacidade de dispersão apresentando boa adaptação ambiental e climática devido a sua característica hibernal (Matzenbacher \& Schneider 2008). A planta por competir com outras forrageiras, causa sérios prejuízos à agricultura e à pecuária, pois se desenvolve tanto em solos cultivados como em áreas com pouca cobertura vegetal ou campos nativos (Sindel 1994).

O impacto econômico de $S$. madagacariensis está relacionado principalmente com seu grau de dispersão e de adaptação em diferentes regiões, e pela competitividade com outras plantas nativas (Sindel 1994). Surtos de intoxicação por $S$. madagascariensis não são frequentes, porém foram descritos no Rio Grande do Sul (Cruz et al. 2010, Stigger et al. 2014). Isto indica a necessidade de combate a esta planta invasora, que aparentemente tem se adaptado bem na região Sul do Estado, principalmente nos municípios de Arroio Grande, Pedro Osório e Capão do Leão a partir do ano 2013, sendo sugerido que a mesma está em pleno processo de adaptação e disseminação nesta região e que outros surtos podem ocorrer nos próximos anos (Stigger et al. 2014).

Os objetivos do presente trabalho foram avaliar métodos de controle de Senecio madagascariensis e Senecio brasiliensis incluindo o uso de ovinos e técnicas de manejo do solo, além de estimar uma lotação adequada de ovinos, conforme o grau de infestação da planta no potreiro, para minimizar os efeitos de seu consumo pelos bovinos.

\section{MATERIAL E MÉTODOS}

\section{Controle de Senecio madagascariensis}

Foram realizados três experimentos em uma propriedade localizada no município do Capão do Leão (S 31ํ4'⒈7" / W 52ํ31'58.3"), invadida por S. madagascariensis que se encontrava em todas as fases vegetativas (brotação, crescimento, floração e sementação) (Fig. 1). Para estimar a infestação dos potreiros utilizou-se um quadrado de madeira de $0,5 \mathrm{~m}^{2}$ que era jogado aleatoriamente no potreiro por 10 vezes, contando-se os pés de plantas a cada lançamento (Quadros 1, 2 e 3). Foram utilizados ovinos da raça Corriedale com dois anos de idade. Antes do início dos experimentos e periodicamente era realizado exame clínico administrando-se tratamento anti-helmíntico quando necessário alternando-se os princípios ativos. 0 manejo dos animais era realizado conforme o utilizado na propriedade, incluindo casqueamento duas vezes por ano, tosquia e banhos para controle de ectoparasitas anualmente. A avaliação das áreas experimentais foi realizada por dois anos por meio de fotografias para a determinação do comportamento da planta e comparação entre as diferentes técnicas de controle.

Biopsias hepáticas nos ovinos experimentais foram realizadas aos 30 e 90 dias após o início de cada experimento. A técnica utilizada foi a percutânea com agulha Menghini modificada descrita por Néspoli et al. (2010). Os ovinos eram mantidos em decúbito lateral esquerdo realizando-se a tricotomia e antissepsia no local da biopsia. Posteriormente a área era infiltrada com $5 \mathrm{ml}$ de solução cloridrato de lidocaína a $2 \%$ nos pontos de introdução da agulha que era inserida em sentido crânio-ventral, no

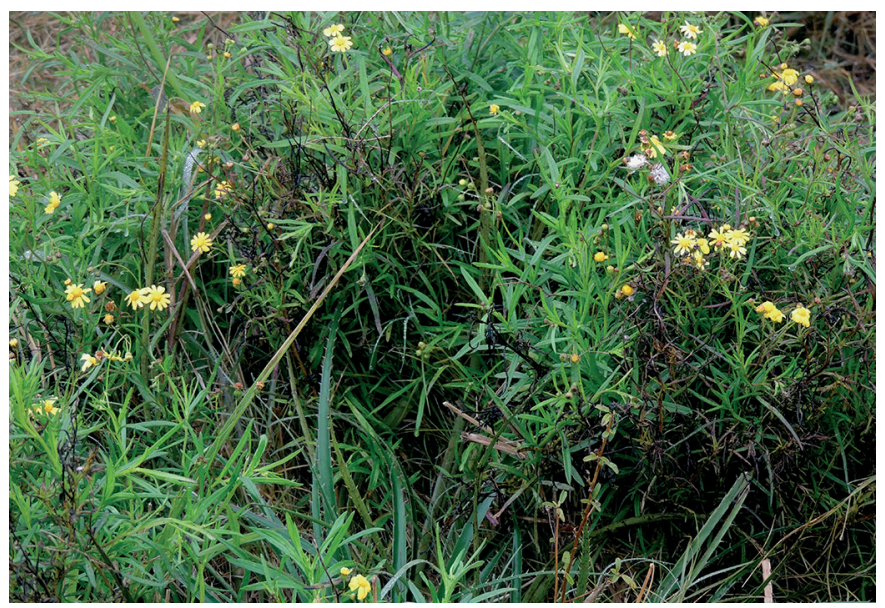

Fig.1. Senecio madagascariensis com flores e sementes. 
Quadro 1. Número de plantas de Senecio madagascariensis em locais de infestação média e alta em uma área utilizada para controle da planta utilizando pastejo com quatro ovinos/ha no primeiro período experimental

\begin{tabular}{|c|c|c|c|c|c|c|c|c|}
\hline \multirow[t]{2}{*}{ Pontos } & \multicolumn{4}{|c|}{$\begin{array}{l}\text { № de plantas } \\
\text { Infestação média }\end{array}$} & \multicolumn{4}{|c|}{$\begin{array}{l}\text { № de plantas } \\
\text { Infestação alta }\end{array}$} \\
\hline & 0 dia & 30 dias & 60 dias & 90 dias & 0 dia & 30 dias & 60 dias & 90 dias \\
\hline 1 & 2 & 0 & 0 & 0 & 7 & 3 & 3 & 0 \\
\hline 2 & 4 & 2 & 0 & 0 & 2 & 2 & 0 & 0 \\
\hline 3 & 2 & 1 & 1 & 0 & 11 & 2 & 0 & 0 \\
\hline 4 & 1 & 0 & 0 & 1 & 10 & 1 & 0 & 1 \\
\hline 5 & 2 & 1 & 1 & 0 & 2 & 0 & 1 & 0 \\
\hline 6 & 1 & 1 & 0 & 0 & 11 & 3 & 0 & 0 \\
\hline 7 & 0 & 0 & 0 & 0 & 9 & 4 & 3 & 0 \\
\hline 8 & 1 & 0 & 0 & 0 & 18 & 2 & 3 & 1 \\
\hline 9 & 4 & 2 & 0 & 0 & 2 & 1 & 1 & 0 \\
\hline 10 & 1 & 2 & 2 & 0 & 2 & 2 & 1 & 0 \\
\hline Total $^{\mathrm{a}} 1$ & $/ 1,5(1$, & $9 / 1(2)$ & $4 / 0(1)$ & $1 / 0(0)^{a}$ & $74 / 8(9)^{\mathrm{c}}$ & $20 / 2(2)$ & $12 / 1(3)$ & $/ 0,2(0) 0,25^{b}$ \\
\hline
\end{tabular}

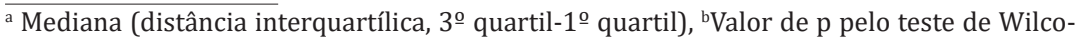
xon $=0,011,{ }^{c}$ Valor de p pelo teste de Wilcoxon $=0,005$.

Quadro 2. Número de plantas de Senecio madagascariensis em locais de infestação média e alta em uma área utilizada para controle da planta utilizando pastejo com quatro ovinos/ha no segundo período experimental

\begin{tabular}{|c|c|c|c|c|c|c|c|c|}
\hline \multirow[t]{2}{*}{ Pontos } & \multicolumn{3}{|c|}{$\begin{array}{l}\text { № de plantas } \\
\text { Infestação média }\end{array}$} & \multicolumn{5}{|c|}{$\begin{array}{l}\text { № de plantas } \\
\text { Infestação alta }\end{array}$} \\
\hline & 0 dia & 30 dias & 60 dias & 90 dias & 0 dia & 30 dias & 60 dias & 90 dias \\
\hline 1 & 1 & 0 & 2 & 0 & 5 & 3 & 0 & 0 \\
\hline 2 & 3 & 2 & 0 & 0 & 3 & 2 & 2 & 0 \\
\hline 3 & 2 & 1 & 0 & 0 & 4 & 2 & 0 & 0 \\
\hline 4 & 1 & 2 & 0 & 0 & 3 & 4 & 0 & 0 \\
\hline 5 & 0 & 1 & 1 & 0 & 4 & 2 & 1 & 0 \\
\hline 6 & 2 & 1 & 1 & 1 & 5 & 3 & 0 & 1 \\
\hline 7 & 2 & 2 & 1 & 0 & 3 & 2 & 0 & 0 \\
\hline 8 & 3 & 2 & 0 & 1 & 4 & 3 & 0 & 1 \\
\hline 9 & 1 & 3 & 2 & 0 & 5 & 4 & 0 & 0 \\
\hline 10 & 2 & 1 & 1 & 0 & 4 & 3 & 1 & 1 \\
\hline Total $^{\mathrm{a}}$ & $/ 2(1,2$ & $15 / 1,5(1$ & $3 / 1(1,25$ & $/ 0(0,25)^{\mathrm{b}}$ & $40 / 4(2)$ & $8 / 3(1,25$ & $4 / 0(1)$ & $3 / 0(1)^{c}$ \\
\hline
\end{tabular}

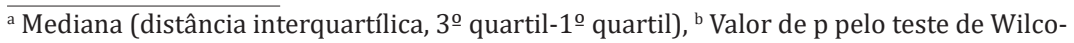
xon $=0,007,{ }^{c}$ Valor de p pelo teste de Wilcoxon $=0,004$

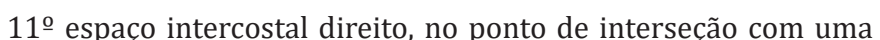
linha imaginária paralela à coluna vertebral, que partia da extremidade lateral da tuberosidade ilíaca. Após ultrapassar a parede torácica e a superfície do fígado, o mandril da agulha era retirado, introduzindo-se a agulha no parênquima hepático por dois a três centímetros. A extremidade externa da agulha era tampada com o dedo indicador, fazendo vácuo e impedindo a perda do fragmento durante a retirada da mesma. Os fragmentos de fígado eram colocados em papel toalha para retirada do excesso de sangue e depois mergulhados em formalina 10\% tamponada.

Experimento 1 (Pastejo contínuo). Foram utilizados 40 ovinos em uma área de 4ha, na qual foram consideradas duas áreas

Quadro 3. Número de plantas de Senecio madagascariensis em locais de infestação baixa, média e alta em uma área para controle da planta utilizando pastejo alternado com 10 ovinos/ha

\begin{tabular}{|c|c|c|c|c|c|c|c|c|c|c|c|c|}
\hline \multirow[t]{2}{*}{ Pontos } & \multicolumn{4}{|c|}{$\begin{array}{c}\text { № de plantas } \\
\text { Parcela A (infestação média) }\end{array}$} & \multicolumn{4}{|c|}{$\begin{array}{c}\text { № de plantas } \\
\text { Parcela B (infestação baixa) }\end{array}$} & \multicolumn{4}{|c|}{$\begin{array}{c}\text { № de plantas } \\
\text { Parcela C (infestação alta) }\end{array}$} \\
\hline & 0 dia & 30 dias $^{a}$ & 90 dias & 150 dias & 0 dia & 30 dias & 90 dias & 150 dias & 0 dia & 30 dias & 90 dias & 150 dias \\
\hline 1 & 1 & 1 & 0 & 0 & 0 & 1 & 0 & 0 & 3 & 5 & 6 & 1 \\
\hline 2 & 2 & 0 & 0 & 0 & 0 & 0 & 0 & 0 & 5 & 2 & 8 & 0 \\
\hline 3 & 1 & 0 & 1 & 0 & 1 & 0 & 1 & 0 & 2 & 3 & 1 & 0 \\
\hline 4 & 0 & 1 & 0 & 1 & 3 & 1 & 0 & 0 & 2 & 1 & 1 & 1 \\
\hline 5 & 2 & 1 & 1 & 0 & 1 & 1 & 1 & 0 & 7 & 4 & 3 & 1 \\
\hline 6 & 1 & 1 & 0 & 0 & 0 & 0 & 1 & 0 & 4 & 3 & 1 & 0 \\
\hline 7 & 2 & 0 & 0 & 0 & 2 & 0 & 0 & 0 & 2 & 1 & 2 & 0 \\
\hline 8 & 1 & 1 & 0 & 0 & 1 & 0 & 0 & 0 & 3 & 3 & 3 & 1 \\
\hline 9 & 1 & 2 & 1 & 0 & 0 & 0 & 0 & 0 & 1 & 3 & 1 & 1 \\
\hline 10 & 1 & 0 & 1 & 0 & 0 & 0 & 0 & 0 & 4 & 5 & 4 & 0 \\
\hline Total $^{\mathrm{b}}$ & $12 / 1(1$ & c $7 / 1(1)$ & $4 / 0(1)$ & $1 / 0(0)^{c}$ & $8 / 0,5(1,25)$ & $3 / 0(1)$ & $3 / 0(1)$ & $0 / 0(0)^{\mathrm{d}}$ & $33 / 3(2,25$ & $30 / 3(2,5)$ & $30 / 2,5(3,5)$ & $5 / 0,5(1)^{\mathrm{e}}$ \\
\hline
\end{tabular}

${ }^{a}$ Pastoreio de 30 dias com intervalos de 60 dias em cada parcela, ${ }^{\mathrm{b}}$ Mediana (distância interquartílica, $3^{\circ}$ quartil-1을 quartil), ${ }^{\mathrm{c}}$ Valor de p pelo teste de Wilcoxon $=0,013,{ }^{\mathrm{d}}$ Valor de p pelo teste de Wilcoxon $=0,039,{ }^{\mathrm{e}}$ Valor de p pelo teste de Wilcoxon $=0,007$. 
no potreiro, uma com alta e outra com moderada infestação seguindo o procedimento de contagem dos pés de planta descrito anteriormente, antes do início do experimento (dia 0) e aos 30, 60 e 90 dias. Os 40 ovinos foram colocados na área em agosto de 2013 permanecendo no local por 90 dias (primeiro período experimental). Na área, após a retirada dos ovinos, o solo estava descoberto e com pouca disponibilidade de forragem e permaneceu sem os ovinos por oito meses. Em abril 2014, após a dessecação da área, foram cultivados, por plantio direto, Lotus corniculatus L. (cornichão), Trifolium repens (trevo branco), Medicago sativa (alfafa) e Festuca arundinacea Schreb. (festuca). Os 40 ovinos foram novamente colocados na área em julho de 2014 (segundo período experimental), permanecendo por 90 dias e a presença da planta foi avaliada nos mesmos intervalos do primeiro período. Os ovinos foram retirados em novembro de 2014 e a área foi avaliada até julho de 2015.

A comparação entre o número de plantas no dia 0 e no dia 90 nos dois anos de observação foi realizada por meio de testes não paramétricos de Wilcoxon, depois de observada a ausência de normalidade na distribuição por testes de Shapiro-Wilk. De forma semelhante foi realizada a comparação entre o número de plantas encontradas ao final do primeiro período experimental de 90 dias com o início do segundo período experimental. Considerou-se o nível de confiança mínimo de $95 \%$ em todas as análises estatísticas. 0 software SPSS 20.0 foi utilizado em todas as análises.

Experimento 2 (Pastejo rotativo em três parcelas). Uma área de campo nativo invadida por S. madagascariensis foi dividida com cerca elétrica em três parcelas de 0,5ha. A infestação em cada parcela (A: moderada, B: baixa, e C: alta) está apresentada no Quadro 3. Foram colocados 10 ovinos em cada área por períodos alternados de 30 dias entre junho e novembro de 2014. Os ovinos foram tratados e manejados da mesma forma que no Experimento 1.

A comparação entre o número de plantas no dia 0 e no dia 150 foi realizada por meio de testes não paramétricos de Wilcoxon, depois de observada a ausência de normalidade na distribuição por testes de Shapiro-Wilk. Considerou-se o nível de confiança mínimo de $95 \%$ em todas as análises estatísticas. 0 software SPSS 20.0 foi utilizado em todas as análises estatísticas.

Após a realização destes experimentos em março de 2015 os ovinos foram retirados da propriedade. A área das parcelas A, B e C foi dessecada em abril para o plantio de pastagem

Experimento 3 (Manejo do solo e cultivo de pastagem). Uma área de campo nativo invadida por $S$. madagascariensis, com média de infestação alta da planta (média $\geq 7,4$ pés) foi acompanhada durante os anos de 2013 e 2014. Nessa área as formas de controlar a disseminação da planta foram determinadas pelo proprietário, sendo utilizadas as técnicas de dessecação, aração e semeadura de pastagem (gramíneas e leguminosas) quando a infestação por S. madagascariensis era considerada alta.

\section{Controle de Senecio spp.}

Experimento 1 (Pastejo contínuo). A partir de levantamento nos protocolos de necropsia do Laboratório Regional de Diagnóstico da Faculdade de Veterinária da Universidade Federal de Pelotas (LRD/UFPel) foi identificada e localizada uma propriedade rural no município de Herval (S 3207'25.6”/WO 53ํㅜ 29'29.7") com histórico de intoxicação por Senecio spp. em bovinos. 0 experimento teve início em março de 2013 e foi finalizado em dezembro de 2014. Nessa propriedade Senecio brasiliensis era a espécie predominante em alta infestação. Foram introduzidos 44 bovinos e 86 ovinos, que permaneceram no local durante um ano. Foram realizadas visitas periódicas trimestrais (abril, julho, outubro e fevereiro), para avaliação da presença e o consumo da planta no potreiro através do acompanhamento visual e fotográfi- co. Realizou-se, ainda, um retorno à propriedade em abril quando o experimento completou um ano. Foi realizada uma roçada após o primeiro trimestre de observação na área infestada onde o solo não continha pedras já que a área geográfica da propriedade é acidentada.

\section{RESULTADOS}

\section{Controle de Senecio madagascariensis}

Experimento 1. Após 90 dias de pastoreio pelos ovinos houve diminuição significativa na quantidade de $S$. madagascariensis nos locais de alta infestação $(\mathrm{p}=0,005)$ (Fig. 2A) e média infestação ( $p=0,011$ ) (Fig. 2B) (Quadro 1). Os ovinos foram retirados e o potreiro passou a ser utilizado pelo proprietário como enfermaria ou como área para manter bovinos por períodos curtos quando da realização de manejo como vacinações ou dosificações. Não foram observadas alterações histológicas nas biopsias hepáticas realizadas nos ovinos.

0 potreiro foi avaliado nos quatro primeiros meses de 2014 e em março ao ser detectada a planta, a área foi dessecada com glifosato (Roundup®), em abril foi roçada e semeada com pastagem. Em junho havia pastagem em abundância e nova infestação por S. madagascariensis (Fig.3). Os 40 ovinos foram recolocados na área por mais 90 dias. Houve diminuição significativa do número de plantas nas duas

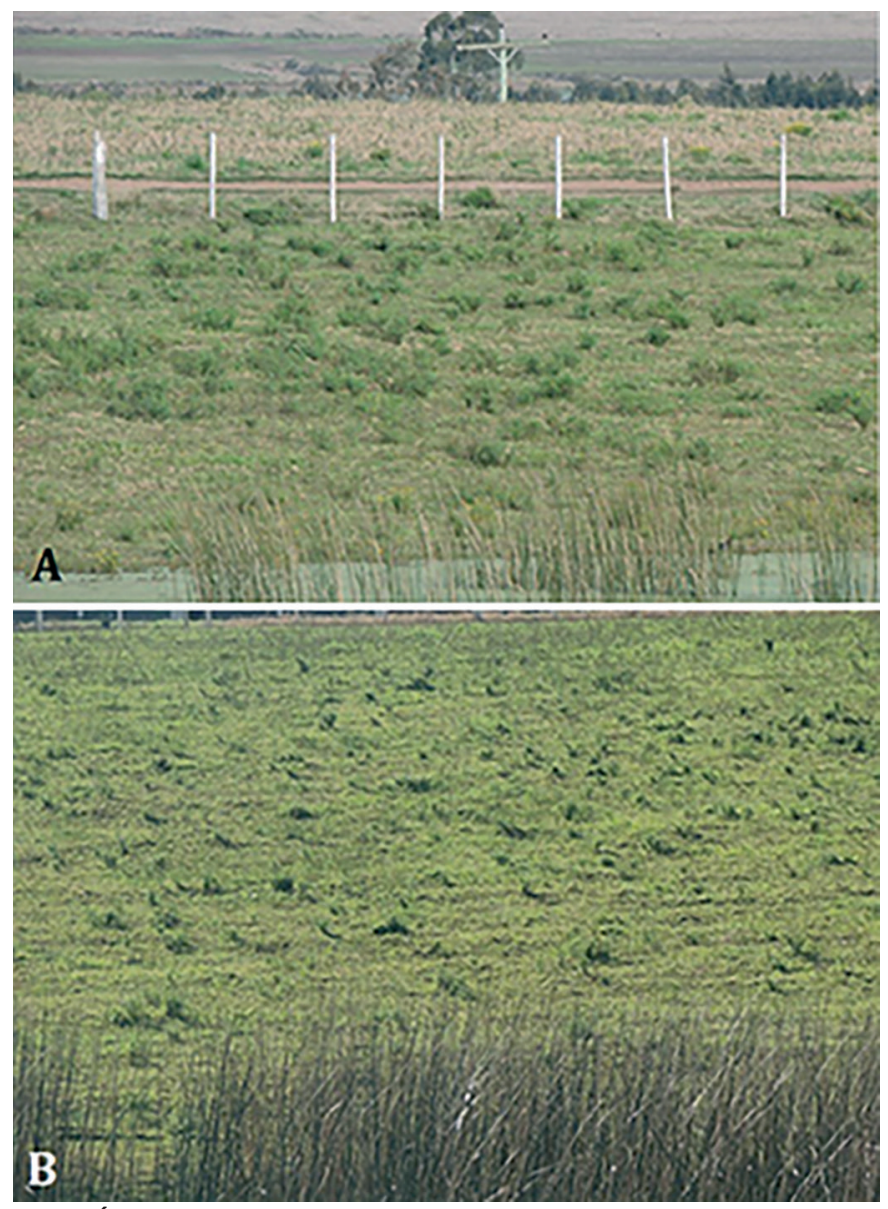

Fig.2. Área infestada por Senecio madagascariensis utilizada para o pastejo contínuo por ovinos durante 90 dias. (A) Área de alta infestação. (B) Área de média infestação. 


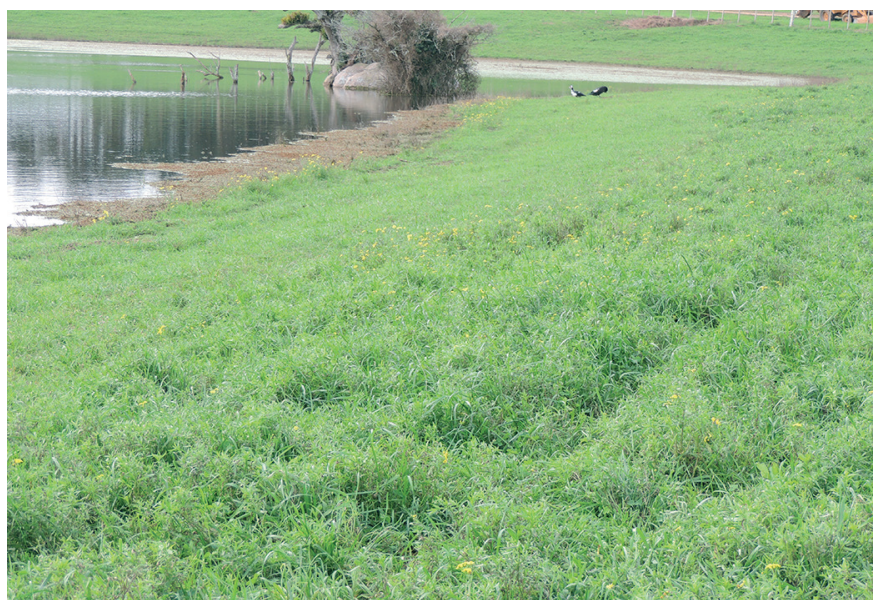

Fig.3. Senecio madagascariensis florescendo na área de infestação alta utilizada no Experimento 1, um ano após o primeiro ingresso dos ovinos.

áreas consideradas de alta e média infestação com valores de $p=0,004$ e $p=0,007$, respectivamente (Quadro2).

A quantidade de plantas observada no dia 90 do primeiro período do Experimento 1 [mediana $=0(\mathrm{DI}=0)$ ] para a quantidade de planta no início do segundo período experimental [mediana $=2(\mathrm{DI}=1,25)]$ foi significativamente maior na área de média infestação $(\mathrm{p}=0,011)$. Do mesmo modo na área de alta infestação no dia 90 do primeiro período experimental [mediana $=0(\mathrm{DI}=0,25)]$ a quantidade de planta era significativamente menor $(\mathrm{p}=0,005)$ que no dia zero do segundo período experimental [mediana $=4(\mathrm{DI}=2)]$ para a quantidade de planta. A média do número de plantas de S. madagascariensis neste período é apresentada no Quadro 2. Em julho de 2015, após nova dessecação havia uma nova pastagem de Trifolium repens (trevo branco), Festuca arundinacea Schreb. (festuca) e Eryngium foetidum (chicória) com infestação acentuada de $S$. madagascariensis após sete meses da retirada definitiva dos ovinos. 0 proprietário passou a arrancar a planta e a quantidade em agosto era mínima. Em outubro havia brotação sem flor misturada a pastagem.

Experimento 2. Controle de Senecio madagascariensis pelo pastejo rotativo utilizando 10 ovinos/0,5ha

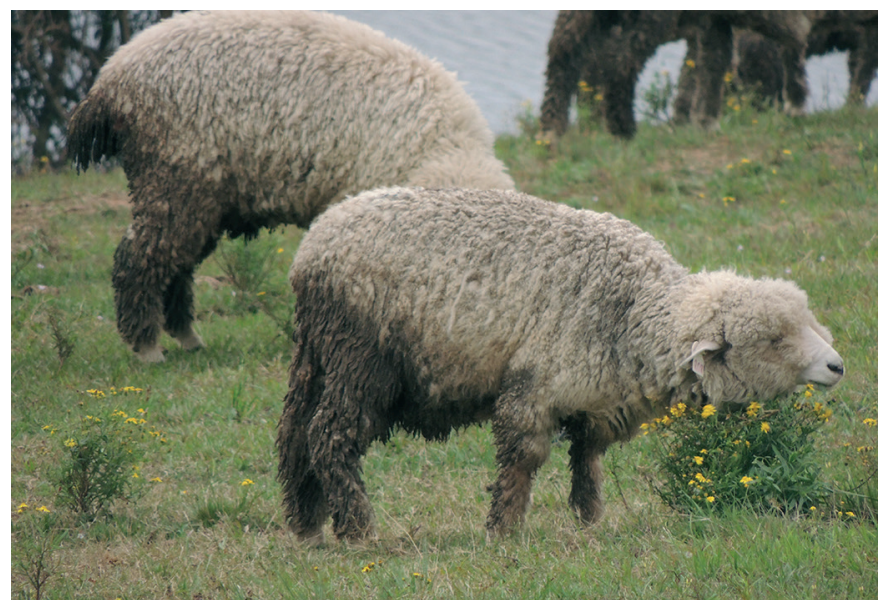

Fig.4. Ovinos consumindo Senecio madagascariensis no Experimento 2 .

(alta lotação). Os ovinos consumiram inicialmente as partes aéreas da planta (Fig. 4), que rebrotava no período de descanso, sendo novamente consumida pelos ovinos no retorno a área (60 dias após). 0 número de plantas presentes nos potreiros durante o experimento é apresentado no Quadro 3. Após a retirada dos animais ao final dos 90 dias intercalados em cada uma das parcelas a planta rebrotou e floresceu em quantidade menor do que no início do experimento e a partir da segunda quinzena de janeiro não foi mais observada na área. A área foi dessecada em abril de 2015 para plantio de pastagem e em agosto do mesmo ano havia brotação da planta em grande quantidade misturada a vegetação (Fig. 5).

A quantidade de planta observada ao final dos 180 dias de experimento foi significativamente menor nas parcelas de média $(p=0,013)$, baixa $(p=0,039)$ e alta $(p=0,007)$ infestação (Quadro 3).

Os ovinos dos dois experimentos não apresentaram lesões histológicas indicativas de intoxicação por alcaloides pirrolizidínicos nas biopsias hepáticas realizadas.

Experimento 3 (Controle de Senecio madagascariensis pelo manejo do solo e cultivo de pastagem). Neste experimento, a partir de novembro de 2013, quando a
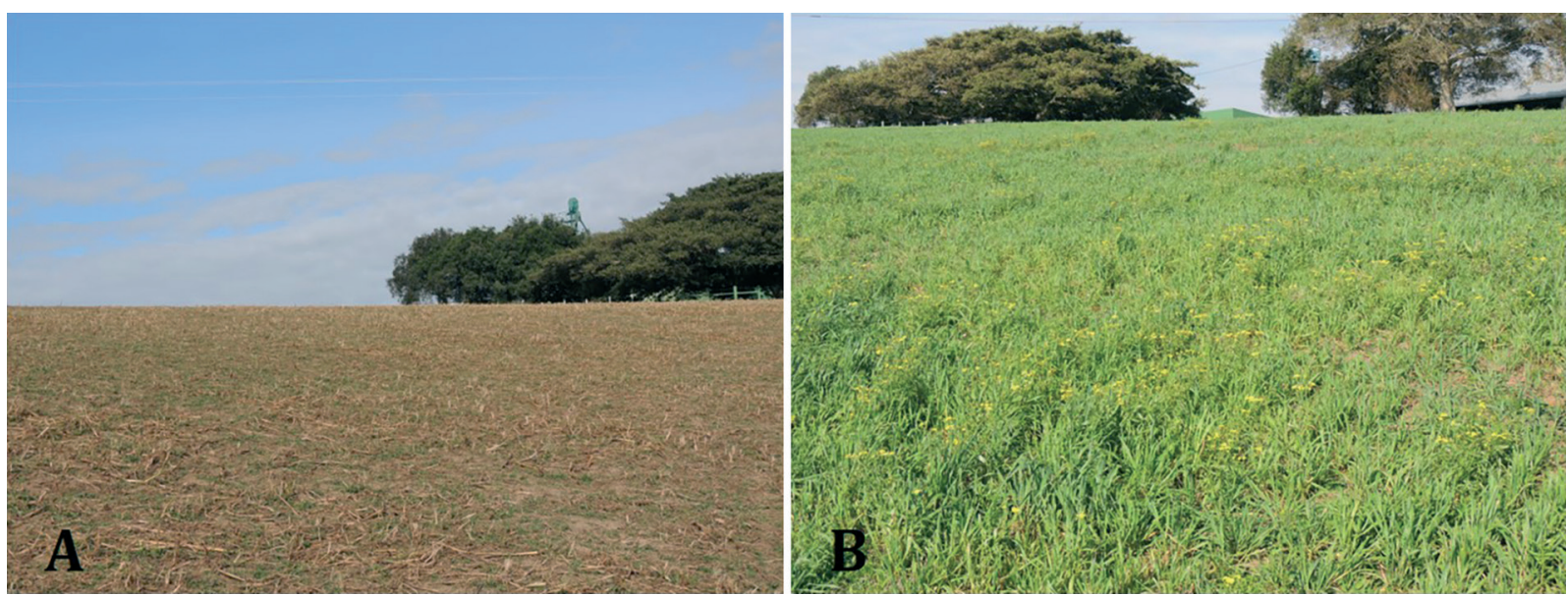

Fig.5. Área do Experimento 2 utilizando pastejo rotativo com ovinos. (A) Área dessecada com Glifosato (Roundup ${ }^{\circledR}$ ) após a retirada dos ovinos (04.2015). (B) Senecio madagascariensis misturado a pastagem, 120 dias após a dessecação (08.2015). 


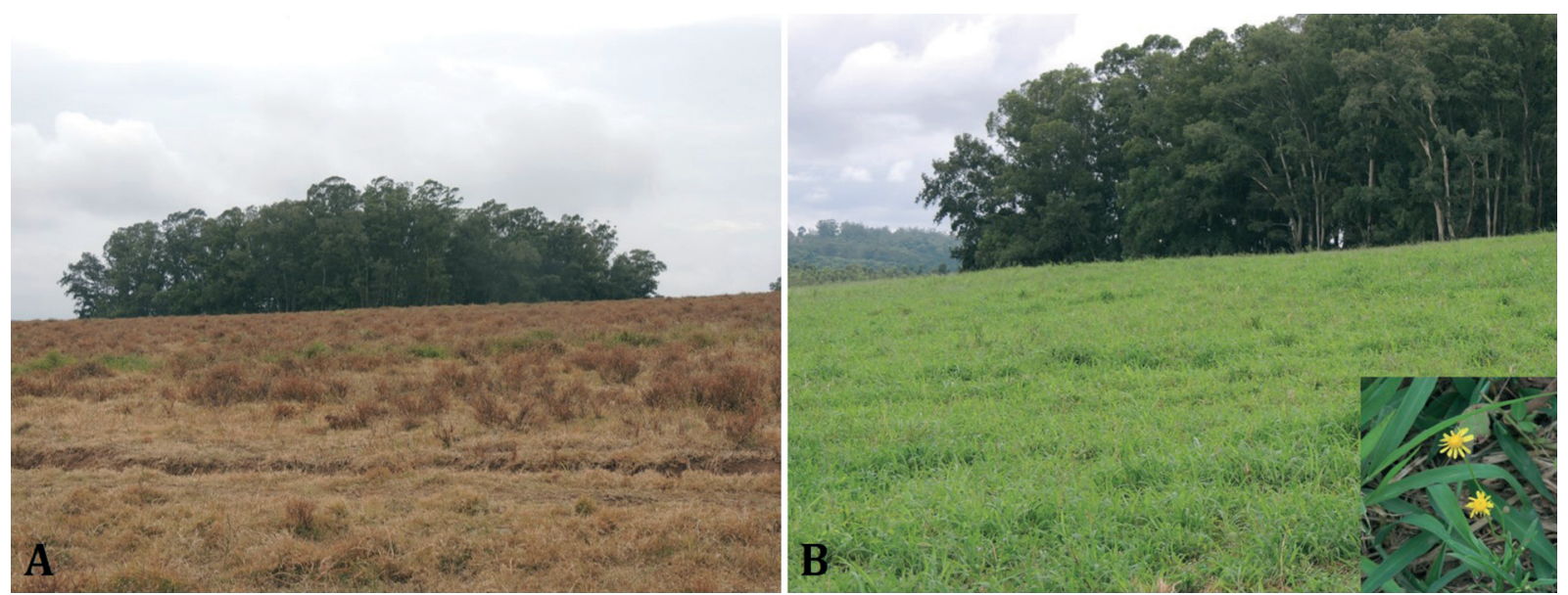

Fig.6. Área do Experimento 3 com utilização de dessecação (10.2013) e plantio de pastagem (12.2013). (A) Área após a dessecação. (B) Senecio madagascariensis brotanto entremeado a pastagem (02. 2014), 120 dias após a dessecação.

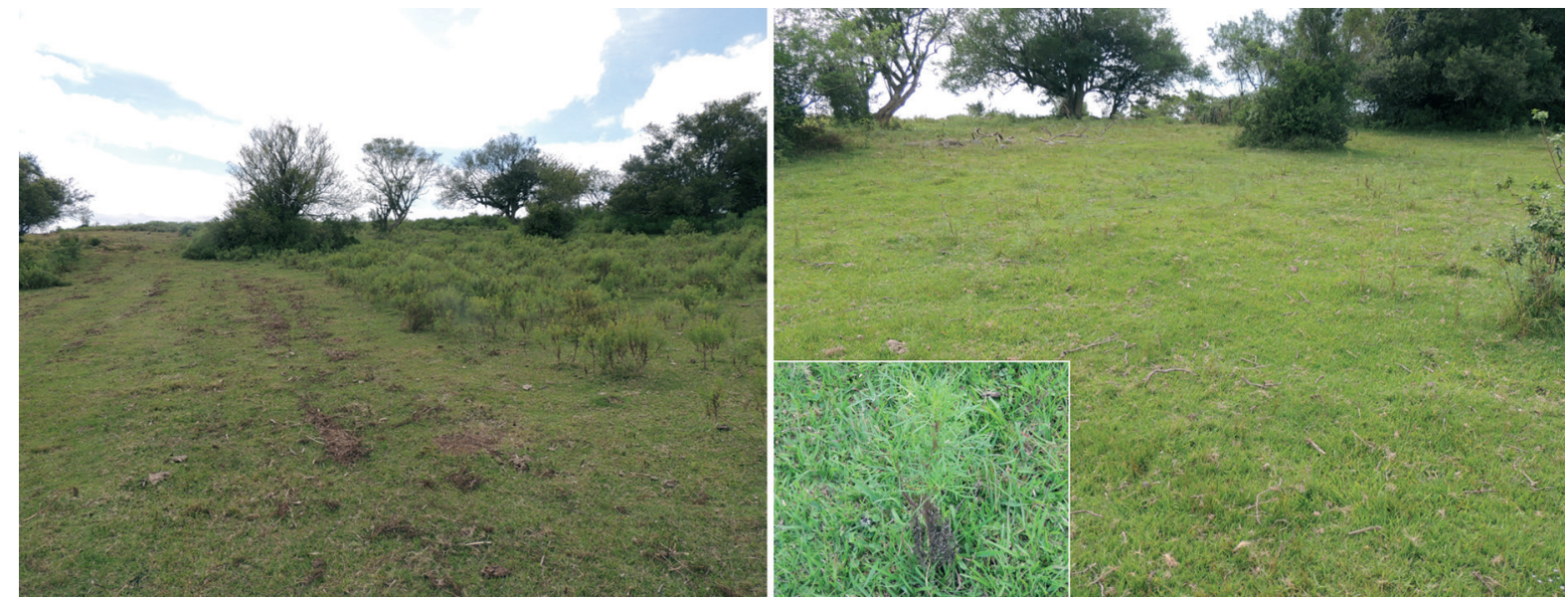

Fig.7. Área do Experimento 4. (A) Observa-se a área roçada (10.2013) ao lado da área com Senecio brasiliensis. (B). Na área roçada há Senecio em brotação (02.2014).

infestação por S. madagascariensis era alta na área foi realizada dessecação com duas aplicações do herbicida glifosato (Roundup $囚$ ) em intervalos de quinze dias. Posteriormente foi feita a aração, a gradagem e o plantio de Panicum spp. e Arachis pintoi (amendoin forrageiro). Em março de 2014 havia crescimento vigoroso da pastagem que apresentava, também, S. madagascariensis em brotação e floração. Em julho de 2014 a área foi roçada. A técnica foi repetida por três vezes uma vez que a planta rebrotava em grande quantidade, após aproximadamente 30 dias da brotação das culturas semeadas (Fig. 6 A e B). A área foi dessecada novamente para o plantio de soja em julho de 2015.

Controle de Senecio brasiliensis com pastoreio contínuo de ovinos em propriedades comercias com histórico de intoxicação em bovinos

Experimento 1. Em março de 2013 a área da propriedade com histórico de intoxicação por Senecio sp. em bovinos foi avaliada visualmente encontrando-se $S$. brasiliensis na fase vegetativa com cerca de um metro de altura e alguns pés secos, além de outras espécies desta planta em pouca quantidade. No primeiro bimestre de avaliação não houve mudança perceptível na quantidade da planta. Nos bimestres subsequentes observou-se que as áreas roçadas apresentaram diminuição na quantidade de pés de $S$. brasiliensis quando comparadas com as áreas onde não foi possível a roçagem (Fig. 7A e B).

\section{DISCUSSÃO}

Os resultados deste trabalho demonstraram a grande dificuldade de controlar $S$. madagascariensis em áreas severamente infestadas, situação já identificada em outros estudos de controle da mesma realizados na Austrália (Sindel et al. 2012) e no Uruguai (Villalba \& Fernadez 2005). Neste último país, foi sugerida a pulverização de áreas invadidas com herbicidas e plantio de pastagens competitivas (Villalba \& Fernadez 2005). Em estudos realizados no Havaí foi recomendado, também, o manejo das pastagens visando estímulo ao seu crescimento e vigor com o uso de fertilizantes e pastejo controlado (Motooka et al. 2004). No presente trabalho, no entanto, ficou demonstrado que o crescimento vigoroso das pastagens semeadas após a dessecação das áreas não impediu a rebrotação da planta, tanto em área com pastejo de ovinos (Experimento 1) como em áreas sem pastejo (Experimento 3).

A utilização de 10 ovinos/ha em pastejo contínuo por 90 dias foi eficiente no controle da planta, ocorrendo diminuição da mesma ao final do período experimental. No 
entanto, observou-se que após a retirada dos animais para plantio de pastagem $S$. madagascariensis estava presente novamente um ano após o primeiro ingresso dos ovinos na área e um ano após o segundo ingresso, mesmo com utilização de herbicida, plantio de pastagem e retirada manual da planta. Ficou evidenciado que os ovinos controlam as partes aéreas diminuindo a sementação sem, contudo, eliminar a planta do solo, que ao ser remexido pela aração favorece a germinação das sementes presentes (banco de sementes do solo - BSS). Tem sido mencionado que a maioria das sementes perde a viabilidade em 3-5 anos. No entanto, experimentalmente foi observado que em ambientes úmidos a maioria das sementes germina até três meses após a dispersão, o que foi observado no presente trabalho. Por outro lado, em condições secas, um pequeno percentual de sementes pode permanecer viável no solo por mais de 10 anos (Sindel \& Coleman 2012). Com base nisto o pastejo intensivo com ovinos por vários anos, de modo a evitar a sementação poderia ser uma prática eficiente a longo prazo para a eliminação da mesma.

Nos experimentos em que o pastejo dos ovinos foi rotativo houve diminuição das plantas provavelmente devido a alta lotação utilizada nas áreas (10 ovinos em 0,5 ha) e a cada retorno dos ovinos a parte aérea em brotação era novamente consumida, impedindo a floração e produção de sementes e por consequência disseminação das mesmas com diminuição gradativa da infestação da área. No entanto, após a retirada definitiva dos ovinos, dessecação e plantio de pastagem a planta voltou a brotar em grande quantidade. Em um trabalho de controle de Senecio spp. os autores concluíram que uma lotação de 0,43 ovinos/ha em pastejo contínuo por 30 dias controlou eficientemente a planta (Soares et al. 2000). A diferença observada na lotação para o controle de $S$. madagascariensis deve-se provavelmente a que, além do ciclo da planta ser mais curto, há vários estágios vegetativos em um mesmo pé, sendo necessária lotação maior para o consumo e controle da planta. Experimentos para o controle de diferentes espécies de Senecio, utilizando dois ovinos por hectare demonstraram que o pastejo interfere nas fases reprodutivas da planta diminuindo a quantidade e o vigor sendo mais eficaz no inverno do que na primavera (Karam et al. 2013). Para o controle de $S$. madagascariensis, no entanto, observou-se que a planta apresentava flores e sementes durante todos os meses do ano, crescendo vigorosamente após o consumo pelos ovinos o que sugere que neste caso é necessário um tempo maior de pastejo e a combinação de outras práticas.

Nos experimentos realizados com pastoreio rotativo foi possível observar que $S$. madagascariensis desaparece das áreas durante o verão, mesmo sem a presença de ovinos, especialmente quando as temperaturas são elevadas e há estiagem prolongada. Por outro lado, tem sido mencionado que nesta situação após as primeiras chuvas há brotação e estabelecimento imediato da planta (Sindel \& Coleman 2012) o que foi observado, também, no presente trabalho. Isto sugere a necessidade de recolocação dos ovinos imediatamente nas áreas para evitar a floração e produção de sementes que ocorre cerca de 6-10 semanas após a germinação (Sindel \& Coleman 2012).
As biopsias hepáticas realizadas demonstraram que os ovinos não apresentaram lesões de seneciose durante o período experimental. Sabe-se que ovinos são mais resistentes aos alcaloides pirrolizidínicos (Craig et al. 1992) e a quantidade do princípio ativo é menor nesta espécie de Senecio (Karam et al. 2011a) confirmando que o uso de ovinos é seguro para o controle da planta. Por sua vez, os bovinos estão sujeitos a intoxicação por $S$. madagascariensis (Cruz et al. 2011, Stigger et al. 2014). 0 controle de $S$. brasiliensis neste trabalho apresentou resultados conflitantes considerando-se que de acordo com estudos anteriores 0,47 ovinos/ha seriam suficientes para controlar a planta (Soares et al. 2000) e no presente estudo foi utilizada uma lotação de 1,04 ovinos/ha, sem obter-se o controle adequado da mesma. Deve considerar-se, no entanto, que provavelmente alguns fatores influíram para que isso ocorresse, tais como a presença da planta com mais de um metro de altura, que dificulta o seu consumo por parte dos ovinos, e a dificuldade de roçagem da área total pela presença de terreno acidentado. Na área roçada, um mês e meio após o início do experimento houve uma diminuição na quantidade de Senecio provavelmente pelo consumo da brotação pelos ovinos, o que não foi observado nas áreas não roçadas. Essa diminuição da planta foi observada, também, em outros estudos de controle de Senecio spp. (Karam et al 2011b).

Nas condições destes experimentos foi possível concluir que o controle de $S$. madagascariensis só poderá ocorrer em uma propriedade rural a longo prazo por meio de pastejo contínuo com no mínimo quatro ovinos por hectare. A dessecação do solo pode eliminar a parte aérea da planta, porém a aração favorece a germinação das sementes presentes no solo. A pastagem, por sua vez, pode competir com $S$ madagascariensis, no entanto, não impede sua germinação. Isto sugere que um conjunto de medidas como pastejo por ovinos, uso de herbicidas e implantação de pastagens nos momentos adequados podem auxiliar no controle da planta minimizando prejuízos econômicos. Sugere-se que o número de ovinos utilizado para o controle de S. brasiliensis deve ser estudado caso a caso dependendo da infestação da área, estágio vegetativo da planta e condições do terreno. No caso de $S$. brasiliensis o controle é aparentemente mais fácil pois a floração e produção de sementes ocorre em épocas mais específicas do ano podendo utilizar-se ovinos nesta época com resultados mais eficientes, especialmente se a prática for associada a métodos complementares.

Agradecimentos.- Trabalho financiado pelo INCT/CNPq Proc.573534/ 2008-0 e CNPq Proc. 304332/2012-7. Agradecemos ao Senhor Heron Krolow e ao Dr. Luis Fernando Gomes pelo empréstimo das áreas em suas propriedades rurais para realização deste trabalho.

\section{REFERÊNCIAS}

Barros C.S., Driemeier D., Pilati C., Barros S.S. \& Castilhos L.M.L. 1992. Senecio spp. poisoning in cattle in Southern Brazil. Vet. Hum. Toxicol. $34(3): 241-246$.

Cabrera A.L. 1963. Flora de la Provincia de Buenos Aires. Compuestas. Buenos Aires: Coleccion Científica del I. N. T. A., 4(6): 433p.

Cabrera A.L. \& Ré R.R. 1965. Sobre un Senecio adventicio en la Provincia de Buenos Aires. Revta Facultad de Agronomía de la Universidad Nacional de La Plata, La Plata, Argentina 41(1):43-50.

Craig A.M., Lathan C.J., Blythe L.L., Schmotzer W.B. \& O’Connor O.A. 1992. 
Metabolism of toxic pyrrolizidine alkaloids from tansy ragwort (Senecio jacobaea) in ovine ruminal fluid under anaerobic conditions. Appl. Environ. Microbiol. 58:2730-2736.

Cruz C.E.F., Karam F.C., Dalto A.C., Pavarini S.P., Bandarra P.M. \& Driemeier D. 2010. Fireweed (Senecio madagascariensis) poisoning in cattle. Pesq. Vet. Bras. 30:10-12.

Grecco F.B., Estima-Silva P., Marcolongo-Pereira C., Soares M.P., Collares G. \& Schild A.L. 2011. Seneciose crônica em ovinos no sul do Rio Grande do Sul. Pesq. Vet. Bras. 31(4):326-330.

Grecco F.B., Schild A.L., Estima-Silva P., Marcolongo-Pereira C., Soares M.P. \& Sallis E.S.V. 2010. Aspectos epidemiológicos e padrões de lesões hepáticas em 35 surtos de intoxicação por Senecio spp. em bovinos no sul do Rio Grande do Sul. Pesq. Vet. Bras. 30(5):389-397.

Ilha M.R., Loretti A.P., Barros S.S. \& Barros C.L. 2001. Intoxicação espontânea por Senecio brasiliensis (Asteraceae) em ovinos no Rio Grande do Sul. Pesq Vet Bras 21:123-138.

Karam F.S.C., Méndez M.C., Jarenkow J.A. \& Riet-Correa F. 2002. Fenologia de quatro espécies tóxicas de Senecio (Asteraceae) na região Sul do Rio Grande do Sul. Pesq. Vet. Bras. 22(1):33-39.

Karam F.S.C., Haraguchi M. \& Gardner D. 2011a. Seasonal variation in pyrrolizidine alkaloid concentration and plant development in Senecio madagascariensis Poir. (Asteraceae) in Brazil, p.179-185. In: Riet-Correa F., Pfister J., Schild A.L. \& Wierenga T. (Eds), Poisoning by Plants, Mycotoxins and related Toxins. CAB International, Wallingford, UK.

Karam F.C., Schild A.L. \& Mello J.R.B. 2011b. Intoxicação por Senecio spp. em bovinos no Rio Grande do Sul: condições ambientais favoráveis e medidas de controle. Pesq. Vet. Bras. 31(7):603-609.

Karam F.S.C., Soares M.P., Haraguchi M., Riet-Correa F., Méndez M.C. \& Jarenkow J.A. 2004. Aspectos epidemiológicos da seneciose na região sul do Rio Grande do Sul. Pesq. Vet. Bras. 24:191-198.

Karam F.C., Moraes J.C.F. \& Schild A.L. 2013. Controle de Senecio spp. Com pastoreio ovino de acordo com sua infestação e fenologia. Pesq. Vet. Bras. 33(9):1109-1115.

Lombardo A. 1983. Flora Montevidensis. Montevideo: Intendencia Municipal de Montevideo, T. 2, p. 274-288.
Matzenbacher N.I. \& Schneider A.A. 2008. Nota sobre a presença de uma espécie adventícia de Senecio (Asteraceae) no Rio Grande do Sul, Brasil. Revta. Bras. Biociênc. 6:111-115.

Méndez M.C. \& Riet-Correa F. 1993. Intoxication by "Senecio tweediei" in cattle in southern Brazil. Vet. Human. Toxicol. 35(1):55.

Méndez M.C. \& Riet-Correa F. 2008. Plantas Tóxicas e Micotoxicoses. 2ª ed. Editora e Gráfica Universitária, Pelotas. 298p.

Motooka P., Nagai G., Onuma K., Duponte M., Kawabata A. \& Fukumoto G. 1999. Control of Fireweed (Senecio madagascariensis). Hawaii Department of Agriculture, and University of Hawaii at Manoa (CTAHR). Weed Control WC-2. of toxic pyrrolizidine alkaloids from tansy ragwort (Senecio jacobaea) in ovine ruminal fluid under anaerobic conditions. Appl. Environ. Microbiol. 58:2730-2736.

Néspoli P.B., Gheller V.A., Peixoto P.V., França T.N., Carvalho A.U., Godoy de Araújo D.K. \& Malm C. 2010. Avaliação de técnicas de biópsia hepática em ovinos. Pesq. Vet. Bras. 30(1):29-36.

Sindel B. \& Coleman M. 2012. Fireweed: a best practice management guide for Australian landholders. University of New England. 33.p.

Sindel B.M. 1994. Impact, ecology and control of the weed Senecio madagascariensis in Australia. In: Caligari P.D.S. \& Hind D.J.N (Eds). Proceedings of the International Compositae Conference, 1994, Kew: The Royal Botanic Gardens, v.2, p. 339-349.

Sindel B.M., Coleman M.J. \& Reeve I.J. 2012. Putting fireweed on the front burner: improving management and understanding 18 th Australasian Weeds Conference (2012) pp. 166-169.

Soares M.P., Riet-Correa F., Méndez M.C., Rosa F.G. \& Carreira E.C. 2000. Controle biológico de Senecio spp. com pastoreio de ovinos. Anais II Reunión Argentina de Patologia Veterinária,l Faculdad de Ciências Veterinárias, Universidad Nacional de La Plata, Ar.

Stigger A.L., Estima-Silva P., Fiss L., Coelho A.C.B., Santos B.L., Gardner D.R., Marcolongo-Pereira C. \& Schild A.L. 2014. Senecio madagascariensis Poir. (Asteraceae): uma nova causa de seneciose em bovinos no Sul do Rio Grande do Sul. Pesq. Vet. Bras. 34(9):851-855.

Villalba J. \& Fernández G. 2005. Otra flor amarilla peligrosa: Senecio madagascariensis Tambo 150:46-48. 